\title{
Características técnicas de bicos de pulverização hidráulicos de jato plano
}

\begin{abstract}
1 DEA/UFV. CEP 36571-000, Viçosa, MG. Fone: (31) 3899-1860. E-mail: jparc@bol.com.br (Foto)
2 DEA/UFV. E-mail: mauri@mail.ufv.br

Protocolo $143-22 / 11 / 2000$

Resumo: A utilização de bicos de pulverização de boa qualidade, que proporcionem cobertura homogênea com espectro de gotas uniforme, é importante para se obter uma aplicação de defensivo agrícola eficiente, sem prejuízos ao meio ambiente. Assim, com este trabalho teve-se, como objetivo, a avaliação das características técnicas de quatro bicos de pulverização hidráulicos disponíveis no mercado, sob diferentes pressões de trabalho e altura da barra porta-bicos, visando-se fornecer subsídios para uma seleção correta de bicos. Em ambiente controlado, determinou-se o perfil de distribuição de cada bico, a vazão, o coeficiente de variação da distribuição volumétrica superficial conjunta dos bicos, o diâmetro mediano volumétrico, o diâmetro mediano numérico, o coeficiente de homogeneidade e a porcentagem de gotas com diâmetro inferior a $100 \mu \mathrm{m}$. De maneira geral, os bicos avaliados apresentaram níveis de uniformidade de distribuição satisfatórios, trabalhando principalmente a pressão de $300 \mathrm{kPa}$ e altura da barra de $50 \mathrm{~cm}$ em relação ao alvo. Os bicos avaliados de acordo com a metodologia utilizada, apresentaram diâmetro mediano volumétrico em torno de $340 \mu \mathrm{m}$ e o espectro de gotas mostrou-se dentro dos padrões recomendados. O coeficiente de homogeneidade do tamanho das gotas foi mais favorável quando se trabalhou a baixas vazões e baixas pressões.
\end{abstract}

João P. A. R. da Cunha ${ }^{1}$ \& Mauri M. Teixeira²

Palavras-chave: gotas, pontas de pulverização, tecnologia de aplicação

\section{Technical characteristics of hydraulic fan spray nozzles}

\begin{abstract}
The use of spray nozzles that provide a homogeneous covering with uniform droplet spectrum is important to obtain an efficient pesticide application, without damage to the environment. Thus, the principal aim of this work was to evaluate the technical characteristics of four hydraulic spray nozzles available in the market, under different working pressures and positions in relation to the objective, seeking to supply indicators for a correct selection of spray nozzles. In a controlled atmosphere, the following parameters were determined: distribution profile, the flow, the coefficient of variation of the nozzle distribution, the volume median diameter, the number median diameter, the homogeneity coefficient, and the percentage of droplets smaller than $100 \mu \mathrm{m}$. In a general way, the nozzles presented satisfactory levels of distribution uniformity, working with a pressure of $300 \mathrm{kPa}$ and $50 \mathrm{~cm}$ of height. The appraised nozzles, in agreement with the used methodology, presented a volume mode diameter of $340 \mu \mathrm{m}$, and the droplets spectrum was shown to be within the recommended patterns. The homogeneity coefficient of the droplet size was better with low flow and low pressure.
\end{abstract}

Key words: droplets, spray nozzles, application technology

\section{INTRODUÇÃO}

Embora desempenhem papel de fundamental importância dentro do sistema de produção agrícola vigente, os fitossanitários têm sido alvo de crescente preocupação por parte dos diversos segmentos da sociedade, em virtude de seu potencial de risco ao meio-ambiente (Barcellos et al., 1998). Cada vez mais se exige, do produtor rural, a utilização correta e criteriosa desses insumos; entretanto, o que se vê no campo é a falta de informação em torno da tecnologia de aplicação. As aplicações podem ser, muitas vezes, eficazes, porém não eficientes, porque não se utilizou da melhor técnica ou equipamento, que determinariam o emprego de menor quantidade de ingrediente ativo na obtenção dos mesmos resultados. Na prática, a dosagem de defensivos empregada é milhares de vezes superior à requerida (Fernandes, 1997).

O objetivo de uma aplicação de defensivo agrícola é colocar o produto no alvo desejado, seja ele uma folha, uma planta 
daninha ou um inseto. Nas décadas passadas, pouco se dava atenção ao tamanho e à uniformidade das gotas produzidas durante a aplicação de produtos fitossanitários, pois o que interessava era molhar bem a cultura, o que se conseguia mediante um volume de calda bastante alto (Carrero, 1996).

Atualmente, entretanto, existe tendência de redução do volume de calda das aplicações fitossanitárias, visando à diminuição dos custos e ao aumento da eficiência da pulverização (Silva, 1999). Esta redução somente é possível quando se dispõe de bicos de pulverização que propiciem uma distribuição transversal uniforme e um espectro de gotas uniforme e de tamanho adequado.

Uma cobertura homogênea pressupõe uma distribuição uniforme, caracterizada por baixos coeficientes de variação da distribuição volumétrica superficial, tanto no sentido longitudinal como no transversal. Esta uniformidade transversal depende do bico utilizado, da sobreposição dos jatos e da posição da barra porta-bicos, em relação ao plano de tratamento (Barthelemy et al., 1990).

Dentro desse contexto, o estudo do espectro de gotas produzidas pelos bicos de pulverização assume grande importância na eficiência de aplicação de fitossanitários, principalmente com o aumento dos problemas fitossanitários associados à deriva e contaminação do lençol freático (Parkin, 1993). Segundo Womac et al. (1999) os fatores que determinam o espectro de gotas produzidas por determinado bico, são vazão nominal, ângulo de descarga, pressão de operação, líquido de aplicação e tipo de bico.

Uma aplicação eficiente exige uma cobertura perfeita da superfície e uma distribuição uniforme das gotas produzidas. No caso de gotas produzidas muito grandes, não haverá uma cobertura perfeita da superfície nem, tampouco, boa uniformidade de distribuição. As gotas muito grandes, devido ao peso, normalmente não aderem às superfícies das folhas, terminando no solo, mas no caso de gotas muito pequenas, geralmente se consegue uma cobertura superficial melhor e uma uniformidade maior de distribuição; entretanto, essas gotas podem evaporar em condições de baixa umidade relativa ou serem levadas pela corrente de ar, provocando o fenômeno da deriva (Teixeira, 1997).

Assim, durante as aplicações de produtos fitossanitários deve-se cuidar para que não apareçam gotas muito grandes nem muito pequenas. Lefebvre (1989) e Márquez (1997) mostram que gotas menores que $100 \mu \mathrm{m}$ são arrastadas com facilidade pelo vento e gotas maiores que $800 \mu \mathrm{m}$ tendem a escorrer da superfície das folhas.

Nos bicos tradicionais, que operam com pressão hidráulica, a formação de gotas é bastante desuniforme e seu tamanho é extremamente desigual dificultando, muitas vezes, uma aplicação eficiente; portanto, torna-se necessário o estudo dos equipamentos de aplicação fitossanitária disponíveis no mercado (Matuo, 1990).

Desta forma, este trabalho teve como objetivo a avaliação das características técnicas de bicos hidráulicos de jato plano, disponíveis no mercado, sob diferentes pressões de trabalho e altura da barra, visando fornecer subsídios para uma correta seleção de bicos de pulverização.

\section{MATERIAL E MÉTODOS}

Os ensaios foram realizados no Laboratório de Mecanização Agrícola do Departamento de Engenharia Agrícola da Universidade Federal de Viçosa. Foram avaliados quatro diferentes bicos de pulverização hidráulicos, tipo leque, com ângulo do jato de $110^{\circ}$, fabricados em poliacetal, adquiridos no mercado: Jacto 110-SF-02, Jacto 110-SF-03, Lurmark 02-F-110 e Lurmark 03-F-110 (Tabela 1). Esses bicos, de acordo com os fabricantes, são de uso geral, produzindo aplicações uniformes quando se sobrepõem as pulverizações, sendo recomendados para trabalhar a $300 \mathrm{kPa}$ de pressão, com altura mínima da barra de $35 \mathrm{~cm}$ em relação ao alvo.

Tabela 1. Especificação dos bicos avaliados

\begin{tabular}{ccc}
\hline $\begin{array}{c}\text { Especificação } \\
\text { do Bico }\end{array}$ & Fabricante & $\begin{array}{c}\text { Vazão Nominal* } \\
\text { (300 kPa de pressão) }\end{array}$ \\
\hline $110-\mathrm{SF}-02$ & $\begin{array}{c}\text { Máquinas Agrícolas } \\
\text { Jacto S/A }\end{array}$ & $0,81 \mathrm{~L} \mathrm{~min}^{-1}$ \\
& $\begin{array}{c}\text { Máquinas Agrícolas } \\
\text { Jacto S/A }\end{array}$ & $1,25 \mathrm{~L} \mathrm{~min}^{-1}$ \\
$110-\mathrm{SF}-03$ & $\begin{array}{c}\text { Lurmark Ltd. } \\
\text { 02-F-110 }\end{array}$ & $0,80 \mathrm{~L} \mathrm{~min}^{-1}$ \\
$03-\mathrm{F}-110$ & Lurmark Ltd. & $1,20 \mathrm{~L} \mathrm{~min}^{-1}$ \\
\hline
\end{tabular}

* Indicada pelo fabricante

Para a caracterização dos bicos, os parâmetros seguintes foram tomados: perfil de distribuição de cada bico, vazão para as pressões de 200, 300 e $400 \mathrm{kPa}$, coeficiente de variação da distribuição volumétrica superficial conjunta dos bicos, diâmetro mediano volumétrico (DMV), diâmetro mediano numérico (DMN), coeficiente de homogeneidade do tamanho das gotas $(\mathrm{CH})$ e porcentagem de gotas com diâmetro inferior a $100 \mu \mathrm{m}$.

Os ensaios de distribuição volumétrica, visando determinar o perfil de distribuição de cada bico e o coeficiente de variação da distribuição conjunta dos bicos, foram feitos utilizando-se os bicos individualmente e em conjunto de três bicos espaçados $50 \mathrm{~cm}$, montados em uma barra porta-bicos sobre uma bancada de ensaios padronizada, de acordo com a norma ISO 5682/1 (ISO, 1986). Durante um tempo mínimo de $60 \mathrm{~s}$, coletou-se o volume do líquido recolhido nas provetas, ao longo da faixa de deposição dos bicos. Trabalhou-se com altura da barra de 30 , 40 e $50 \mathrm{~cm}$ em relação à bancada e pressões de pulverização de 200,300 e $400 \mathrm{kPa}$.

Para o estudo do espectro de gotas, utilizou-se um equipamento de pulverização costal, a pressão constante $\left(\mathrm{CO}_{2}\right)$. As análises do espectro de gotas foram realizadas a partir das impressões das gotas recolhidas em etiquetas de plástico PVC, com dimensões de 3 por $6 \mathrm{~cm}$. Para se obter melhor visualização das gotas e maior contraste entre as gotas e as etiquetas, utilizou-se um corante preto dissolvido na água de pulverização. As etiquetas se situaram ao longo da faixa de aplicação. Trabalhou-se com pressões de 200 e $300 \mathrm{kPa}$ e altura do bico em relação às etiquetas amostradoras de $50 \mathrm{~cm}$. Após a passagem do pulverizador, a uma velocidade de $4 \mathrm{~km} \mathrm{~h}^{-1}$, as etiquetas foram imediatamente fotografadas em equipamento digital, para posterior análise, mediante o programa de análise de imagens "Image Tool". Determinou-se o DMV, o DMN, o $\mathrm{CH}$ e a porcentagem de gotas com diâmetro inferior a $100 \mu \mathrm{m}$. 
Optou-se pela presente metodologia devido à sua facilidade de reprodução, a nível de campo.

Os ensaios foram realizados em ambiente controlado, com o intento de minimizar o efeito das condições ambientais. Os manômetros utilizados foram previamente calibrados por meio de um manômetro padrão, obtendo-se a relação entre pressão indicada e pressão real.

Para a análise estatística dos dados de uniformidade de distribuição volumétrica superficial e de espectro de gotas, considerou-se o experimento em delineamento inteiramente casualizado, com três repetições. Utilizou-se o teste de Tukey, em nível de $5 \%$ de probabilidade, para o estudo comparativo das médias dos tratamentos.

\section{RESULTADOS E DISCUSSÃO}

\section{Uniformidade de distribuição}

Os bicos de pulverização, trabalhando isoladamente, proporcionam um perfil característico que depende de sua vazão nominal, ângulo de abertura, altura da barra sobre o alvo e qualidade de fabricação, dentre outros fatores. Este perfil deve ser considerado para se fazer as recomendações de utilização dos bicos, de maneira a se obter uma cobertura uniforme; assim, os ensaios da distribuição volumétrica superficial mostraram que: os bicos 110-SF-02 apresentaram ligeira assimetria no perfil, com pequena depressão na zona central, e irregularidades a baixa pressão; os bicos 02-F-110 também apresentaram ligeira assimetria, com depressão na zona central; os bicos 110-SF-03 apresentaram perfil mais uniforme, com pequenas irregularidades a pressões menores; os bicos 03-F-110 também apresentaram perfil uniforme, com pequena depressão na zona central. Na Figura 1 tem-se o perfil de distribuição volumétrica

A. Bico 110-SF-02

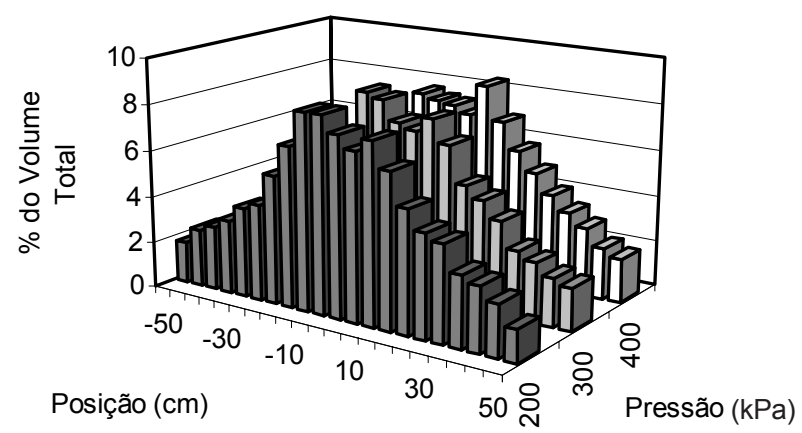

B. Bico 02-F-110

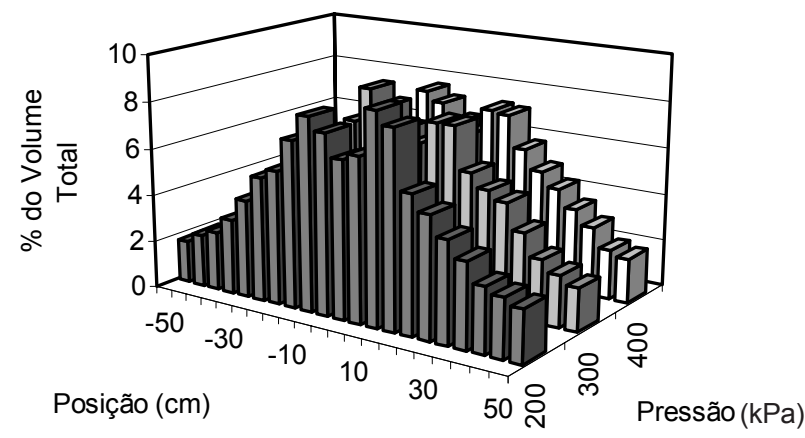

de cada bico trabalhando isoladamente a $50 \mathrm{~cm}$ de altura, em diferentes pressões.

$\mathrm{Na}$ Tabela 2 tem-se as vazões, em $\mathrm{L} \mathrm{min}^{-1}$, dos bicos avaliados, nas diferentes pressões, e a influência da altura da barra porta-bicos e da pressão de trabalho sobre o coeficiente de variação médio (\%) da distribuição volumétrica do conjunto de bicos. É possível perceber-se aumento da vazão com o aumento da pressão, conforme indicação dos fabricantes.

Tabela 2. Vazão dos bicos e influência da altura da barra portabicos e da pressão de trabalho, sobre o coeficiente de variação médio (\%) da distribuição volumétrica do conjunto de bicos

\begin{tabular}{|c|c|c|c|c|c|}
\hline \multirow{3}{*}{ Bico } & \multirow{3}{*}{$\begin{array}{c}\text { Pressão } \\
(\mathrm{kPa})\end{array}$} & \multirow{3}{*}{$\begin{array}{c}\text { Vazão } \\
\left(\mathrm{L} \min ^{-1}\right)\end{array}$} & \multicolumn{3}{|c|}{ Coeficiente de Variação (\%) } \\
\hline & & & \multicolumn{3}{|c|}{ Altura $(\mathrm{cm})$} \\
\hline & & & 30 & 40 & 50 \\
\hline \multirow{3}{*}{ 110-SF-02 } & 200 & 0,695 & $19,8 \mathrm{Aa}$ & $10,0 \mathrm{Ba}$ & $8,9 \mathrm{Ba}$ \\
\hline & 300 & 0,845 & $17,4 \mathrm{Ab}$ & $8,5 \mathrm{Bb}$ & $7,8 \mathrm{Bb}$ \\
\hline & 400 & 0,950 & $15,5 \mathrm{Ac}$ & $8,4 \mathrm{Bb}$ & $8,3 \mathrm{Bab}$ \\
\hline \multirow{3}{*}{$110-\mathrm{SF}-03$} & 200 & 1,060 & $22,0 \mathrm{Aa}$ & $9,6 \mathrm{Ba}$ & $7,4 \mathrm{Ca}$ \\
\hline & 300 & 1,290 & $12,1 \mathrm{Ab}$ & $8,5 \mathrm{Ba}$ & $5,7 \mathrm{Cb}$ \\
\hline & 400 & 1,450 & $10,9 \mathrm{Ac}$ & $8,9 \mathrm{ABa}$ & $6,7 \mathrm{Bab}$ \\
\hline \multirow{3}{*}{$02-\mathrm{F}-110$} & 200 & 0,690 & $16,4 \mathrm{Aa}$ & $14,4 \mathrm{Ba}$ & 7,2 Cab \\
\hline & 300 & 0,820 & $14,7 \mathrm{Ab}$ & $9,2 \mathrm{Bb}$ & $6,7 \mathrm{Cb}$ \\
\hline & 400 & 0,930 & $16,7 \mathrm{Aa}$ & $8,9 \mathrm{Bb}$ & $8,8 \mathrm{Ba}$ \\
\hline \multirow{3}{*}{$03-\mathrm{F}-110$} & 200 & 1,040 & $19,3 \mathrm{Aa}$ & $8,7 \mathrm{Ba}$ & $5,5 \mathrm{Ca}$ \\
\hline & 300 & 1,200 & $17,1 \mathrm{Ab}$ & $6,8 \mathrm{Bb}$ & $5,4 \mathrm{Ca}$ \\
\hline & 400 & 1,380 & $17,2 \mathrm{Ab}$ & $6,0 \mathrm{Bb}$ & $5,6 \mathrm{Ba}$ \\
\hline
\end{tabular}

* Valores seguidos pela mesma letra maiúscula e minúsculas, nas linhas e colunas para cada bico, não diferem a nível de $5 \%$ de probabilidade, segundo teste de Tukey

Na Figura 2 tem-se a avaliação da uniformidade de distribuição do conjunto de bicos, sob a forma de coeficiente de variação.

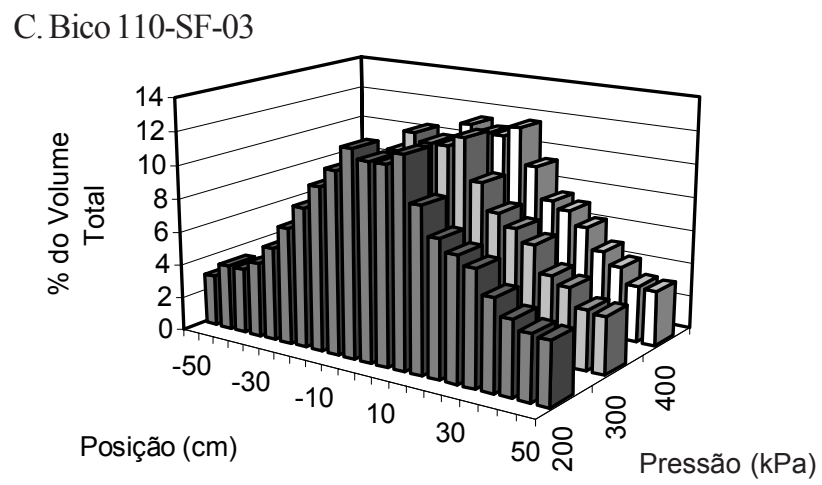

D. Bico 03-F-110

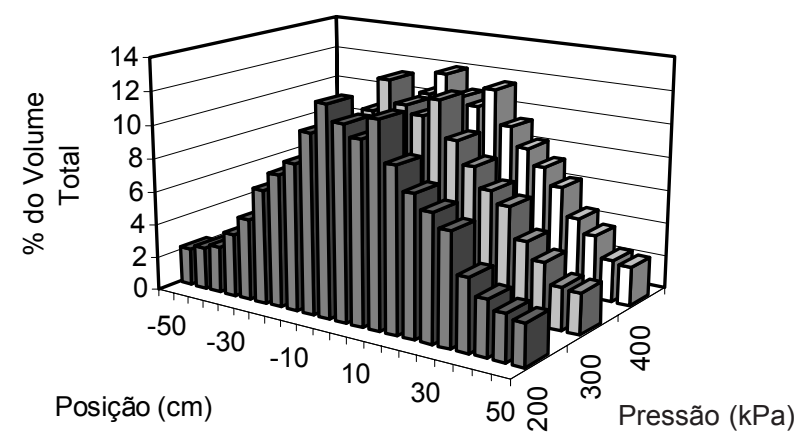

Figura 1. Perfil de distribuição volumétrica dos bicos avaliados, trabalhando isoladamente a $50 \mathrm{~cm}$ de altura, em diferentes pressões 
A. Bico 110-SF-02

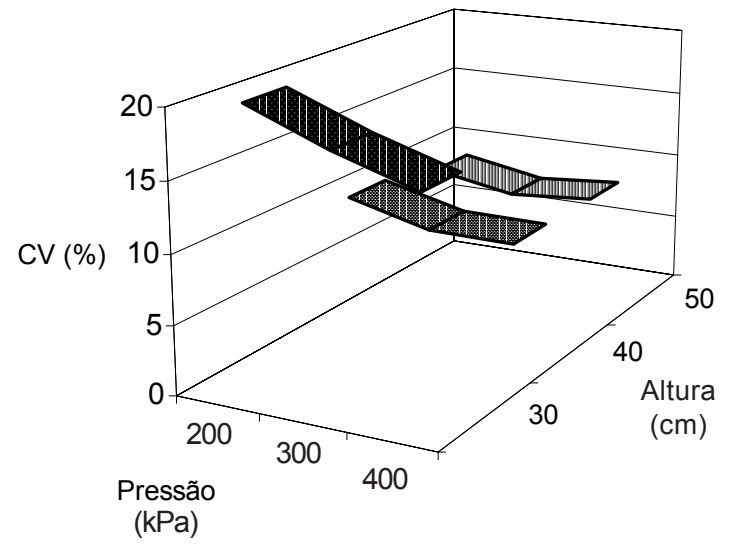

B. Bico 02-F-110

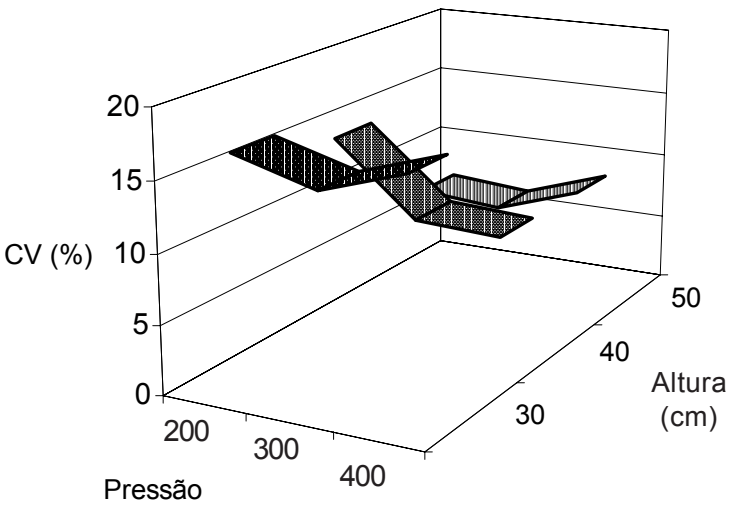

$(\mathrm{kPa})$
C. Bico 110-SF-03

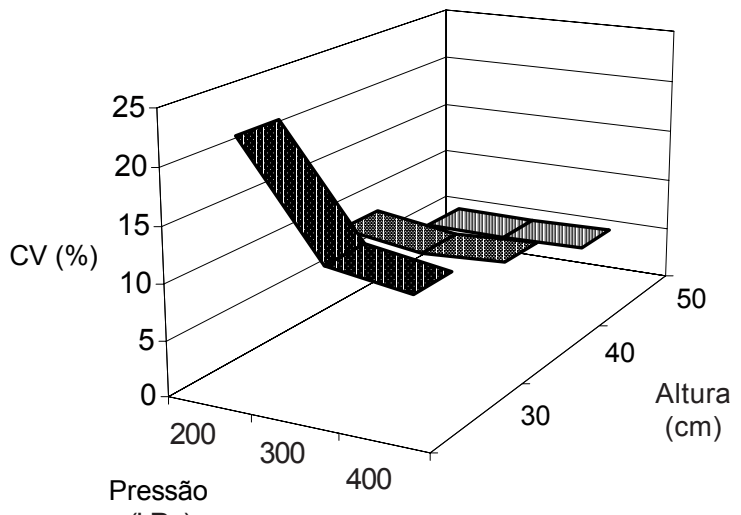

$(\mathrm{kPa})$

D. Bico 03-F-110

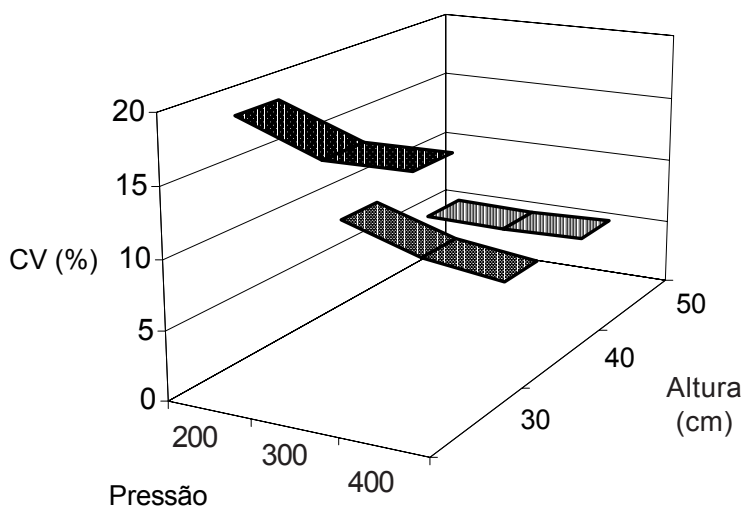

$(\mathrm{kPa})$

Figura 2. Influência da altura da barra porta-bicos e da pressão de trabalho sobre o coeficiente de variação médio da distribuição volumétrica do conjunto de bicos avaliados

Analisando-se os resultados, é possível verificar-se que as melhores condições de trabalho, para os quatro bicos estudados, ocorreram quando os mesmos operaram a $300 \mathrm{kPa}$ de pressão e $50 \mathrm{~cm}$ de altura da barra.

É importante avaliar os dados, levando-se em conta que, no caso específico do Comitê Europeu de Normalização, por exemplo, o coeficiente de variação máximo admitido é de 7,0\% para a altura de barra e pressão indicada pelo fabricante, e 9,0\% para as demais alturas e pressões (Norma prEN 12761-2, ECS, 1997). Portanto, não é recomendado trabalhar-se com os bicos avaliados a uma altura de $30 \mathrm{~cm}$ em relação ao alvo, pois a sobreposição dos jatos não é suficiente para se obter boa uniformidade de distribuição; além disso, não se recomenda trabalhar com os bicos 110-SF-02 e 02-F-110 a pressão de $200 \mathrm{kPa}$ e altura da barra de $40 \mathrm{~cm}$ em relação ao alvo.

\section{Espectro de gotas}

Em todos os ensaios sobre o estudo da população de gotas, utilizaram-se etiquetas plásticas para a amostragem, as quais permitiram identificar-se as impressões das gotas, mesmo trabalhando com os bicos de maior vazão.

Os valores obtidos do espectro de gotas dos bicos avaliados, trabalhando a pressão de 200 e $300 \mathrm{kPa}$, encontram-se na Tabela 3. É possível verificar-se que o coeficiente de homogeneidade do tamanho das gotas $(\mathrm{CH})$ foi mais favorável quando se trabalhou com baixas vazões. Os valores obtidos variaram de 2,4 a 3,3, encontrando-se dentro da faixa aceitável para bicos do tipo leque que, de acordo com Carrero (1996) se situa entre 2 e 5.
Tabela 3. Espectro de gotas ${ }^{*}$ dos bicos avaliados

\begin{tabular}{|c|c|c|c|c|}
\hline Bico & $\begin{array}{l}\mathrm{DMN} \\
(\mu \mathrm{m})\end{array}$ & $\begin{array}{l}\text { DMV } \\
(\mu \mathrm{m})\end{array}$ & $\mathrm{CH}$ & $\begin{array}{c}\% \text { Gotas Menores } \\
\text { que } 100 \mu \mathrm{m}\end{array}$ \\
\hline \multicolumn{5}{|c|}{ A. Pressão de $200 \mathrm{kPa}$} \\
\hline 110-SF-02 & $125 \mathrm{a}$ & $330 b$ & $2,6 b$ & $36 a$ \\
\hline 110-SF03 & $128 \mathrm{a}$ & $395 a$ & $3,1 \mathrm{a}$ & $34 \mathrm{a}$ \\
\hline 02-F-110 & $125 \mathrm{a}$ & $300 \mathrm{c}$ & $2,4 b$ & $34 \mathrm{a}$ \\
\hline 03-F-110 & $128 \mathrm{a}$ & $405 a$ & $3,2 \mathrm{a}$ & $30 \mathrm{a}$ \\
\hline \multicolumn{5}{|c|}{ B. Pressão de $300 \mathrm{kPa}$} \\
\hline 110-SF-02 & $104 \mathrm{a}$ & $290 b$ & $2,8 b$ & $38 \mathrm{a}$ \\
\hline 110-SF03 & $108 \mathrm{a}$ & $352 \mathrm{a}$ & $3,2 \mathrm{a}$ & $36 a$ \\
\hline $02-\mathrm{F}-110$ & $108 \mathrm{a}$ & $280 \mathrm{~b}$ & $2,6 b$ & $37 \mathrm{a}$ \\
\hline $03-\mathrm{F}-110$ & $110 \mathrm{a}$ & $360 \mathrm{a}$ & $3,3 \mathrm{a}$ & $32 \mathrm{a}$ \\
\hline
\end{tabular}

À medida que se deseja trabalhar com volumes menores de calda, deve-se exigir mais da qualidade dos bicos e, especialmente, da homogeneidade do espectro de gotas. É importante que, para esta finalidade, os bicos de pulverização tenham coeficientes de homogeneidade próximos a 2, para variação de pressão de 100 a $500 \mathrm{kPa}$.

Os diâmetros da mediana volumétrica (DMV) que variaram de 280 a $405 \mu \mathrm{m}$, também se encontraram dentro dos valores recomendados para os bicos do tipo leque. De acordo com Márquez (1997) o valor médio do DMV para esse tipo de bico, trabalhando-se a pressão de $300 \mathrm{kPa}$, é de $300 \mu \mathrm{m}$. Desta forma, evitam-se problemas de escorrimento que, geralmente, ocorrem 
com gotas maiores que $800 \mu \mathrm{m}$, e problemas de deriva, que acontecem com gotas menores que $100 \mu \mathrm{m}$.

Os valores percentuais de gotas com diâmetro inferior a 100 $\mu \mathrm{m}$ servem como informações complementares para avaliar a susceptibilidade das gotas à deriva e evaporação. Percebe-se que parte do líquido pulverizado está susceptível à deriva e evaporação: para a pressão de $200 \mathrm{kPa}$, em média, $34 \%$ das gotas possuem diâmetro inferior a $100 \mu \mathrm{m}$ e, para a pressão de $300 \mathrm{kPa}, 36 \%$. A medida em que se aumenta a pressão, o risco de deriva e evaporação também aumenta; entretanto, trabalhando-se com bicos de maior vazão, tende-se a diminuir este problema.

\section{CONCLUSÕES}

Nas condições em que os ensaios foram conduzidos, os resultados permitiram as seguintes conclusões:

1. A uniformidade de distribuição volumétrica superficial dos bicos avaliados, representada pelo coeficiente de variação, foi influenciada pela vazão nominal, pressão de trabalho e altura de trabalho da barra.

2. Os bicos avaliados apresentaram, de maneira geral, níveis de uniformidade de distribuição satisfatórios, trabalhando principalmente a pressão de $300 \mathrm{kPa}$ e altura da barra de $50 \mathrm{~cm}$ em relação ao alvo.

3. O espectro de gotas dos bicos avaliados mostrou-se dentro dos padrões recomendados. O coeficiente de homogeneidade do tamanho das gotas foi mais favorável quando se trabalhou com baixas vazões e baixas pressões.

\section{LITERATURA CITADA}

Barcellos, L.C.; Carvalho, Y.C.; Silva, A.L. Estudo sobre a penetração de gotas de pulverização no dossel da cultura da soja [Glycine max. (L.) Merrill]. Engenharia na Agricultura, Viçosa, v.6, n.2, p.81-94, 1998.
Barthelemy, P.; Boisgointer, D.; Jouy, L.; Lajoux, P. Choisir les outilis de pulverisation. Paris: Institut Technique des Céréales et des Fourrages, 1990. 160p.

Carrero, J.M. Maquinaria para tratamientos fitosanitarios. Madrid: Mundi-Prensa, 1996. 159p.

ECS - European Committee for Standardization. Agricultural and forestry machinery - Sprayers and liquid fertilizer distributors - Environmental protection - Part 2: Low crop sprayers - prEN 12761-2:1997. Brussels: CEN, 1997. 17p.

Fernandes, H.C. Aplicação de defensivos agrícolas: Teoria da gota. Engenharia na Agricultura, Viçosa, 1997. 14p. Caderno Didático 24

ISO - International Organization for Standardization. Equipment for crop protection, ISO standards 5682/1/1981. Geneva: ISO, 1986. p.358-371.

Lefebvre, A.H. Atomization and sprays. International Series: Combustion. New York: Hemisphere Publishing Corporation, 1989. 421p.

Márquez, L. Tecnología para la aplicación de defensivos agrícolas. In: Congresso Brasileiro de Engenharia Agrícola, 26, 1997, Campina Grande. Anais... CD-Rom. Palestra.

Matuo, T. Técnicas de aplicação de defensivos agrícolas. Jaboticabal: UNESP/FUNEP, 1990. 139p.

Parkin, C.S. Methods for measuring spray droplet sizes. In: Matthews, G.A.; Hislop, E.C. (eds.). Application technology for crop protection. Wallingford: CAB International, 1993. p.57-84.

Silva, O.C. Tecnologia de aplicação de fungicidas. In: Canteri, M.G.; Pria, M.D.; Silva, O.C. (eds.). Principais doenças fúngicas do feijoeiro. Ponta Grossa: UEPG, 1999. p.127-137.

Teixeira, M.M. Influencia del volumen de caldo y de la uniformidad de distribución transversal sobre la eficacia de la pulverización hidráulica. Madrid: UPM-ETSIA, 1997.310p. Tesis Doctoral

Womac, A.R.; Maynard, R.A.; Kirk, I.W. Measurement variations in reference sprays for nozzle classification. Transactions of the ASAE., St. Joseph, v.42, n.3, p.609-616, 1999. 